\title{
Study Based on Mobile Ad Hoc Routing Protocols in Control System
}

\author{
Yan Zhao ${ }^{1 *}$ and Tao Peng ${ }^{2}$ \\ ${ }^{1}$ School of Measurement and Communication, Harbin University of \\ Science \&Technology, Harbin, 150080, P. R. China \\ ${ }^{2}$ Star Fiberglass Harbin Co, Ltd, Harbin, 150060, P. R. China \\ 1,2 jazhao1111@163.com
}

\begin{abstract}
A mobile ad hoc network is a multi-hop, centerless and ad hoc network consisting of a group of mobile terminals with wireless communication transceiver devices, and it does not require the support of fixed facilities, composed of a large number of dispersed wireless nodes. Each node can act as both a terminal and as a router, which means that each node can serve as a terminal to produce or receive data information and also serve as a router to forward the data information from other nodes. As the mobile terminals are mostly battery-operated and extremely sensitive to energy consumption, it is very important to prolong the lifetime of the network by reducing the energy consumption of the node. In this paper, a mobile ad hoc network is introduced briefly first, followed by the analysis of the research status of the AODV protocol for the mobile ad hoc network, with focus placed on the introduction to a routing protocol MTTC-ADOV (Minimum Total Transmission on Cost AODV) that can optimize energy consumption by choosing the routing path involving the minimum total transmission cost from all possible routing sets, with consideration given to power and node energy consumption as the routing selection goal. The total transmission cost includes the energy consumption of nodes on the link during transmission, energy consumption of nodes during the message receiving process and energy consumption of nodes during the message transmission process.The MTTC$A O D V$ protocol required the minimum transmission power of each hop on the multi-hop link consisting of the routing, thereby extending the network lifecycle.
\end{abstract}

Keywords: mobile ad hoc routing protocol; MTTC-AODV protocol-based energy consumption optimization

\section{Introduction}

With the constant development and maturity of wireless communication technology, people have increasingly strict requirements for flexible, easy and fast ways of communication, which has greatly stimulated the rapid development of wireless communication networks. Mobile ad hoc network technology, as a new network technology, is rapidly penetrating into each civil communication field. A mobile ad hoc network, referred to as an ad hot network or MANET, is a temporary multi-hop autonomous system consisting of a group of mobile terminals (also known as mobile nodes) with wireless transceiver devices [1]. AODV protocol is an on-demand routing control protocol specially designed for MANET and it is one of the few protocols that have been approved by IETF as protocols for RFC documents [2]. This paper involves introducing the AODC protocol mechanism and putting forward an energy consumption optimization routing protocol MTTC-AODV intended for AODC protocol. 


\section{ADOC Protocol -based Optimization Program MTTC-AODV}

\subsection{Classifications and Common Topological Structures of MANET Routing Protocols}

MANET routing protocols can have different classifications based on different angles, as shown in Figure. 2-1. Based on the angle of routing discovery, MANET routing protocols can be divided into active routing protocols and on-demand routing protocols [3]; based on the logical topology form adopted by the network, MANET routing protocols can be divided into plane routing protocols and hierarchical routing protocols; based on the factor involving the use of a GPS system as an auxiliary condition of routing, MANET routing protocols can be divided into geographic positioning routing protocols and non- geographic positioning routing protocols.

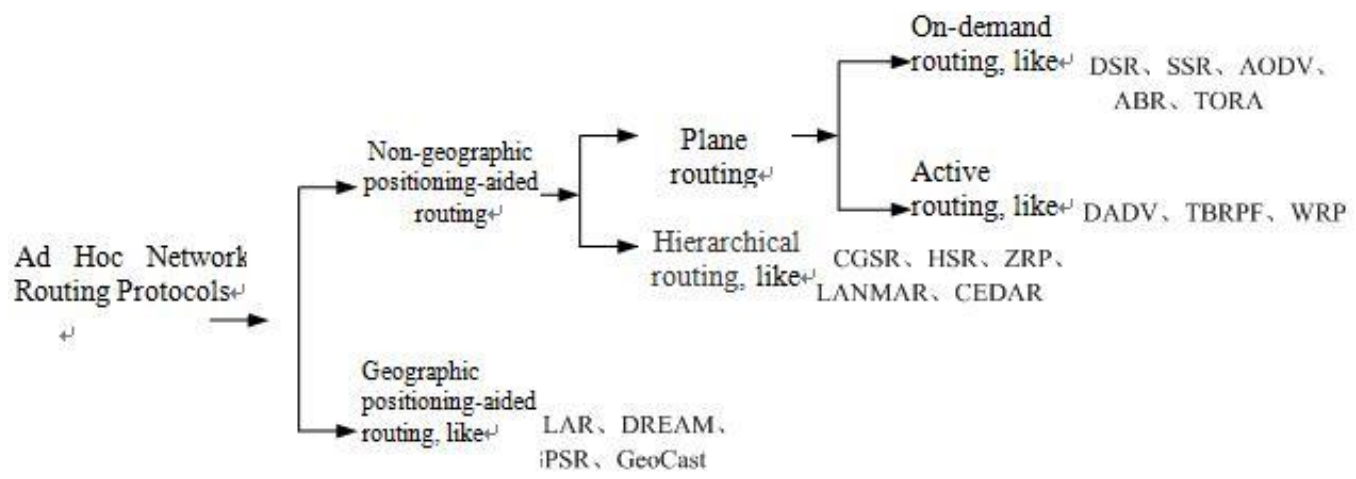

Figure 2-1. Classification of Routing Protocols

The topological structures to MANET [4] are generally divided into star networks, mesh networks and hybrid networks (hierarchical networks), as shown in Figure. 2-2. MANET generally features a large number of network nodes, large density, frequent dynamic network structure changes and complexity. Star topology is selected in this paper.
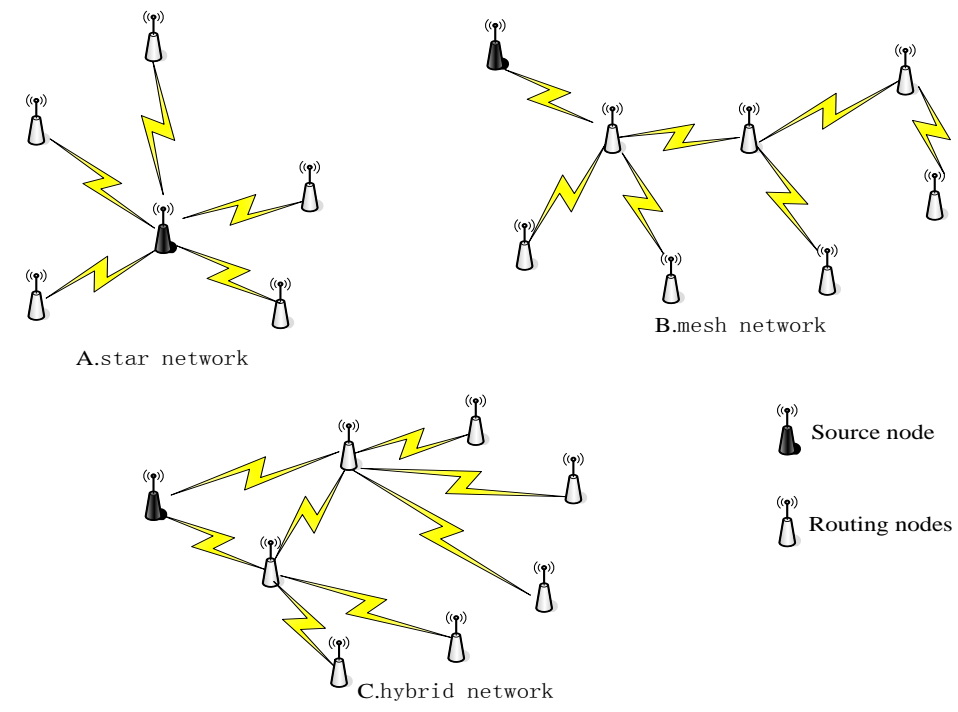

Figure 2-2. Common Topological Structures in MANET 


\subsection{Mechanism of AODV Protocol}

AODV (On-Demand Distance Vector Routing) protocol [2] uses a routing discovery mechanism based on destination nodes. The source node broadcasts a RREQ packet,and the nodes receiving the RREQ packet create reverse routing to the source node.The destination node responds to the RREQ packet after receiving it,and the reply packet transmits to the source node in a unicast way and establishes forward routing to the destination node along the way.

AODV protocol includes route discovery, route maintenance and route repair. When the source node needs to communicate with a certain destination node, it first searches the table of routes maintained at this node for a route to the destination node.If any, it will immediately use this route to transmit a data packet and generates a RREP packet,or it will broadcast RREQ packet to all neighbors to start a route discovery process to find a route available to reach the destination route.After RREQ is generated,corresponding nodes process and forward the RREQ and give RREP.The RREQ and RREP transmission in the route discovery process is as shown in Figure. 2-3.
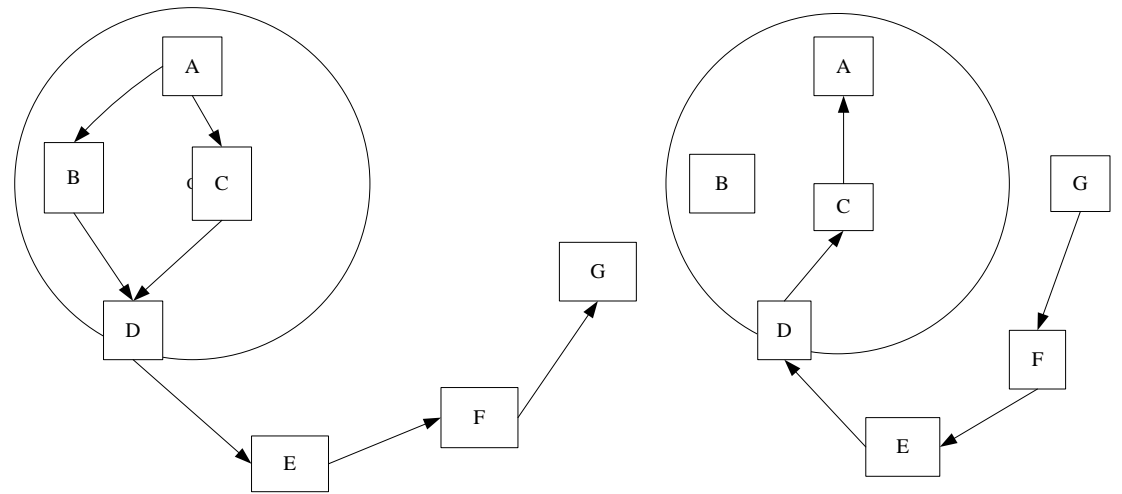

Figure 2-3. RREQ and RREP Transmission in the Route Discovery Process

The left part in Figure.2-3 is an example showing the transmission of a RREQ data package, where the circular area represents the wireless signal transmission range of Node C. When there exists an effective route from the Source Node A to Destination Node G, AODV protocol doesn't work. It is possible to send data to Destination Node G through direct unicasting. When there exists no effective route from the Source Node A to Destination Node G, a route search and establishment process will start.Source Node A broadcasts a RREQ, including such parameters as source node address, source node sequence number, destination source address, destination node sequence number and hop count.When Middle Nodes B, C, D, E and F receive the RREQ, they create or update the reverse routes to Source Node A and continue to broadcast the RREQ message. The right part in Figure.2-3 shows that when Destination Node G receives the RREQ, it sends RREP to Source Node A, and the RREP reaches Source Node A after being forwarded by several middle nodes, based on which route establishment is confirmed, during which a forward route is established. RREQ is transmitted along several path, but RREP is transmitted to the source node along the shortest route $(\mathrm{A} \rightarrow \mathrm{C} \rightarrow \mathrm{D} \rightarrow \mathrm{E} \rightarrow \mathrm{F} \rightarrow \mathrm{G})$, i.e. selection of route involving the shortest time dimension.

ADOV uses a distributed routing mode based on the table of routes and in the route maintenance process, when the route is not used any more, the node will delete the corresponding entries from the table of routes. At the same time, the node will monitor the state of the next hop node in an active node. In the table of routes, it is necessary to record the contents shown in Table 2-1 for each entry [5]. Management of sequence numbers is 
the key to preventing routing loops. In case of a broken link, it is possible to make route entries invalid by increasing sequence numbers and metric (hop count).

Table 2-1. Contents of ADOV Protocol Route Table

\begin{tabular}{|c|c|}
\hline Destination IP Address & Next Hop \\
\hline Destination Sequence No. & List of Precursors \\
\hline Interface & Life time \\
\hline Hop Count & Routing Flags \\
\hline Last Hop Count & \\
\hline
\end{tabular}

\subsection{Energy Consumption Optimization-based AODV Protocol Improvement}

Currently, most routing protocols for MANET are based on the minimum hop count, and this routing method has a good effect on a wired network. However, due to no consideration given to the issue concerning node energy, the traditional protocols for MANET are not suitable for ad hoc networks. In this paper, AODV routing protocol has been improved, an energy consumption optimization program is put forward, and the improved routing protocol is called MTTC-AODV (the minimum total transmission cost AODV) [6].

The MTTC-AODV algorithm requires the minimum transmission power of each hop on the multi-hop link composing the route and the minimum energy consumption in the message transmission process, avoiding the unbalanced energy consumption of each node during route selection and route failure caused by too large node energy, thereby prolonging network lifecycle.

The MTTC-AODV algorithm adds a new field,Total $\mathrm{T} \times$ Cost, to the traditional AODV routing protocol and RREQ packet. The function of the new field is to record and calculate the cumulative sum of the cost of all nodes on the RREQ transmission path, as shown in Fig. 2-4. Source Node s sends a RREQ packet at the minimum cost, and when its neighboring nodes receive the RREQ, MTTC-AODV will calculate the minimum transmission cost of the link (,$n)$. Node $n$ will be accumulated into the Total $\times$ Cost of the request packet and will continue to forward the RREQ at the minimum cost.

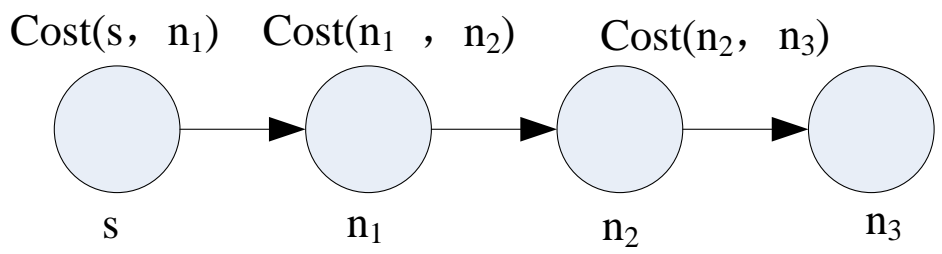

Figure 2-4. Total $\times$ Cost Value of Node n3

In Figure 2-4, $\operatorname{Cost}(\mathrm{s}, \mathrm{n} 1)$ denotes the total cost for data transmission from Source Node $\mathrm{s}$ to Node $\mathrm{n} 1, \operatorname{Cost}(\mathrm{n} 1, \mathrm{n} 2)$ denotes the total cost for data transmission from Node $\mathrm{n} 1$ to Node $\mathrm{n} 2$, and $\operatorname{Cost}(\mathrm{n} 2, \mathrm{n} 3)$ denotes the total cost for data transmission from Node $\mathrm{n} 2$ to Node $n 3$. The RREQ of Source Node $s$ reaches Node $n 3$ via Nodes $n 1$ and $n 2$, in which case the Total $\mathrm{T} \times \operatorname{Cost}$ value in the RREQ equals the sum of $\operatorname{Cost}(\mathrm{s}, \mathrm{n} 1), \operatorname{Cost}(\mathrm{n} 1, \mathrm{n} 2)$ and $\operatorname{Cost}(n 2, n 3)[7]$. 


\subsection{System Model}

A mobile network model consists of a large number of mobile nodes that are randomly distributed, with a wireless link used for communication, with such mobile nodes forming a temporary multi-hop autonomous system. At any time, a random topological structure is formed between nodes through wireless channels, and nodes are able to move randomly, which may cause the change in the network topology [8]. An ad hoc network can either operate in an independent environment or connect to the existing network infrastructure through the gateway, with its typical basic network mode as shown in Figure 2-5.

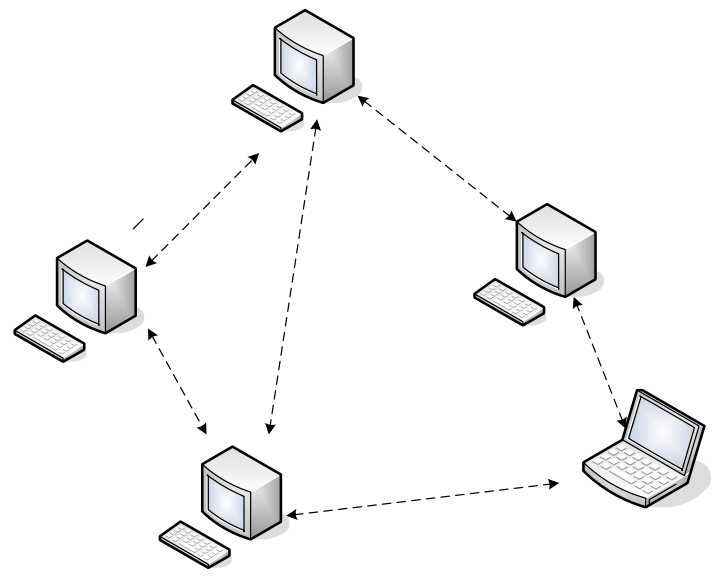

Figure 2-5. Network Model

Considering high-level application, in a node model adopted in MTTC-AODV protocol, a node contains a number of node behavior modules, each of which describes the behavior of some nodes, such as data generation, storage, processing, path selection and transmission. We will analyze the advantages of MTTC-AODV protocol in terms of network performance through simulation.

\section{Simulation Analysis}

\subsection{Simulation Environment}

In order to analyze the superiority of MTTC-AODV protocol that can optimize energy consumption, Matlab simulation is used to construct a simulation platform, which provides many functions such as professional word processing, symbol computing, control implementation and visualized modeling and simulation besides its outstanding numerical computing capacity. A network algorithm can be used to conduct experimental simulation to evaluate its performance.

In this experimental simulation, the nodes are distributed on the experimental platform through random function and the transmission radius of sensor nodes is determined based on the actual network transmission radius. The energy of each node in the network can be random or a set value can be given to initial energy. The network simulation transmission area is set to be a $100 \mathrm{~m} \times 100 \mathrm{~m}$ square area, with 20 to 100 nodes randomly distributed, with specific simulation experimental parameters as shown in Table 3-1. 
Table 3-1. Input Parameter Comparison Table

\begin{tabular}{|l|l|l|l|}
\hline $\begin{array}{l}\text { Experimental } \\
\text { Parameter }\end{array}$ & Parameter Value & $\begin{array}{l}\text { Experimental } \\
\text { Parameter }\end{array}$ & Parameter Value \\
\hline Simulation Area & $100 \mathrm{~m} \times 100 \mathrm{~m}$ & Number of Nodes & $20-100$ \\
\hline $\begin{array}{l}\text { Communication } \\
\text { Radius }\end{array}$ & $0-50 \mathrm{~m}$ & $\begin{array}{l}\text { Number of } \\
\text { Simulations }\end{array}$ & 20 times \\
\hline $\begin{array}{l}\text { Energy of Source } \\
\text { Node }\end{array}$ & No Limit & $\begin{array}{l}\text { Energy of Source } \\
\text { Node }\end{array}$ & $100-1000 \mathrm{~J}$ \\
\hline $\begin{array}{l}\text { Number of Source } \\
\text { Node }\end{array}$ & 1 & Node Distribution & Random \\
\hline
\end{tabular}

\subsection{Simulation Result Analysis}

In the simulation realization process, in case the simulation environment is the same as the parameters, the optimized MTTC-AODV protocol is compared with the AODV protocol that has not been optimized, with the following figures obtained through simulation.

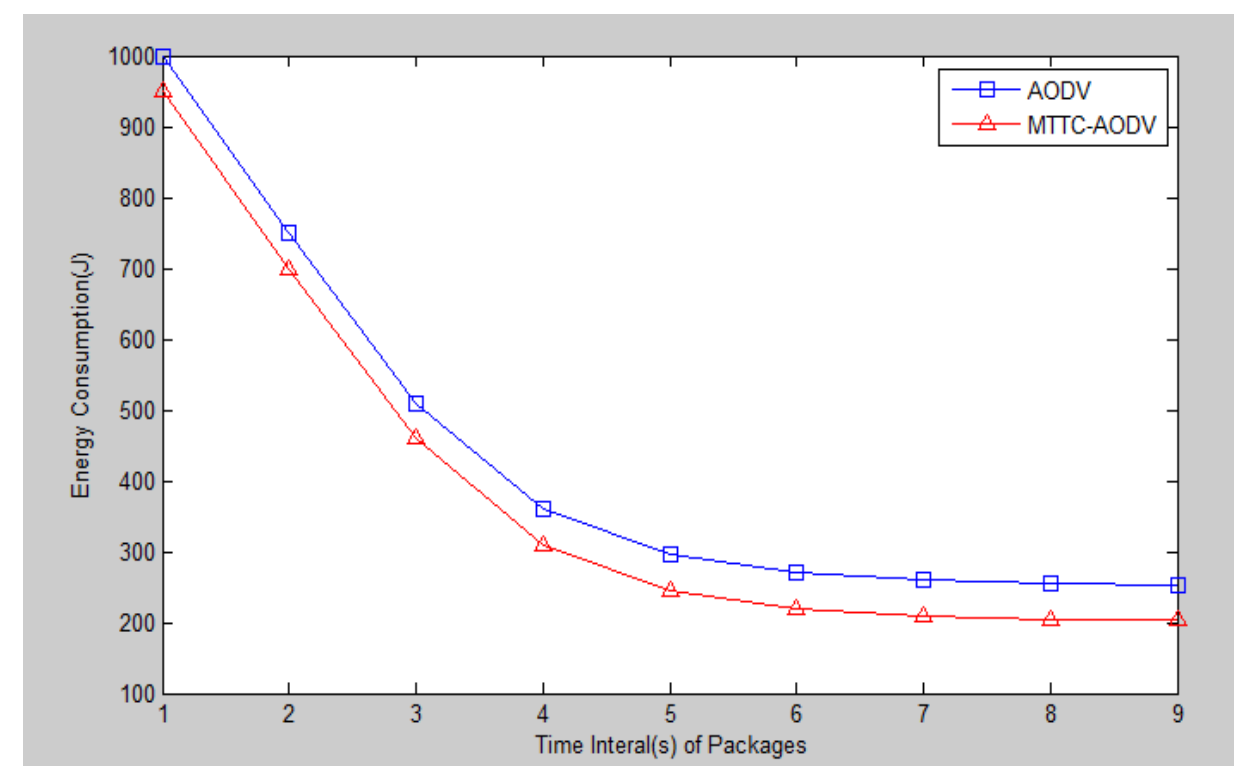

Figure 3-1. Energy Consumption Simulation Diagram

Node energy consumption refers to the total energy consumed during the transmission of a certain number of data packages from the sensor node to the destination node. It can be seen through simulation result comparison that due to the consideration given to the minimum total transmission cost, the optimized MTTC-AODV protocol has obtained a good result, compared with AODV protocol. With the increase in the data arrival interval, the energy consumed by the whole system model is on the gradual decrease.

Node power consumption reduction and further extension of network lifecycle are the key issues involved in MANET and also an important indicator for measuring MTTCAODV protocol. The network lifetime diagram is as shown in Figure 3-2. 


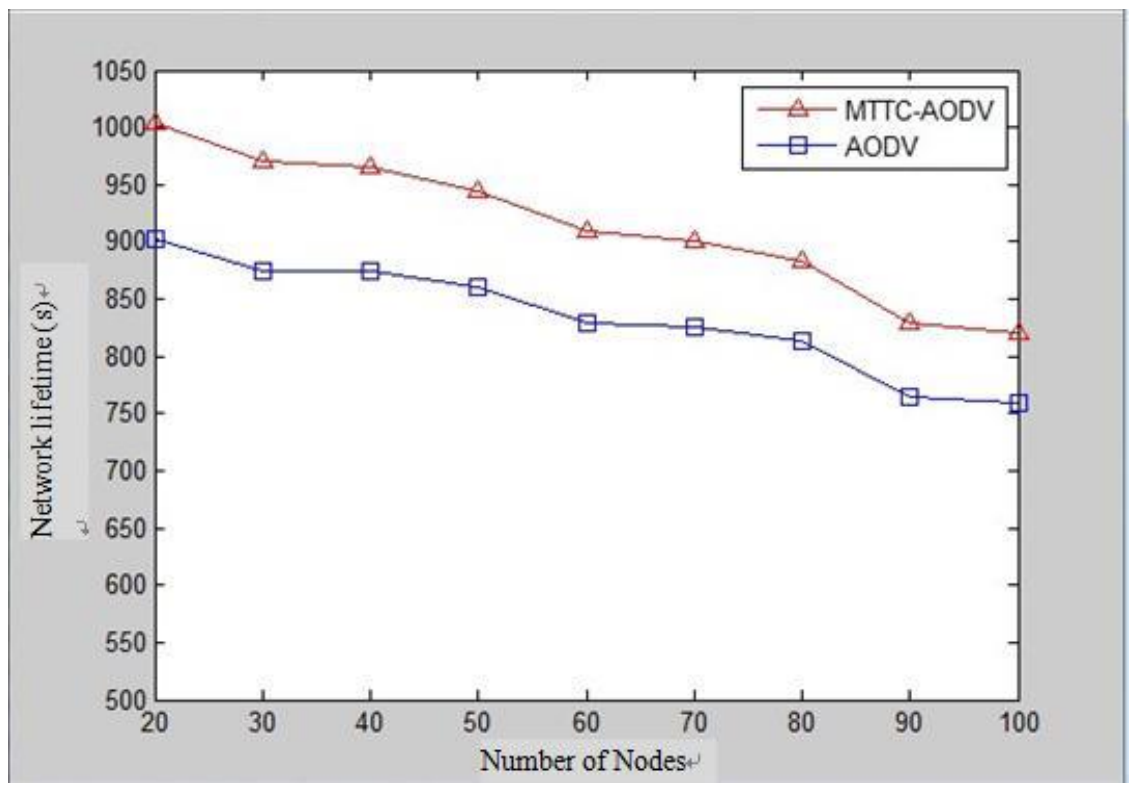

Figure 3-2. Lifetime Simulation Diagram

In the AODC protocol, the increase in the number of nodes, channel competition and increased signal interference in its lifetime have contributed to a higher transmission failure rate, resulting in rapid energy consumption, while the optimized MTTC-AODV protocol gives additional consideration to the energy consumption of nodes, avoiding large energy consumption of nodes caused by a high transfer rate and frequent use of nodes as middle forward nodes, striving to maximize the network lifetime. Figure. 3-3 shows the performance of the MTTC-AODV protocol based on different energy consumptions due to different numbers of nodes. It can be seen in Figure 3-3 that when the number of network nodes is between 65 and 75, the AODV and MTTC-AODV protocols show the minimum energy consumption.

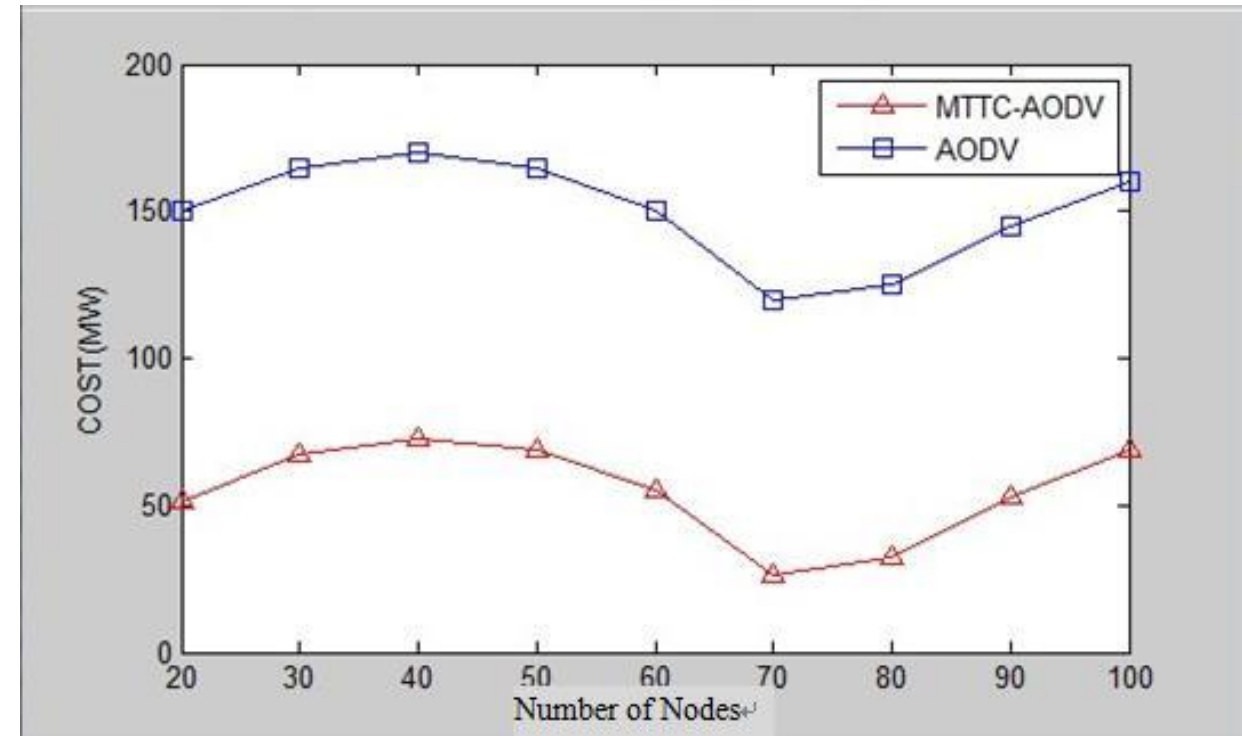

Figure 3-3. Energy Consumption Diagram of Different Nodes 


\section{Conclusions}

In this paper, the most popular AODV routing protocol for MANET is analyzed, and based on the features of low power consumption of MANET, optimization and improvement are implemented in terms of energy consumption reduction. The optimized MTTC-AODV protocol has effectively reduced the energy consumption of nodes and increased the lifetime of the whole network. Theoretical analysis and energy consumption simulation experiment show that compared with the AODC protocol, the optimized MTTC-AODV protocol has great advantages in terms of node energy consumption reduction, especially suitable for MANET.

\section{Acknowledgements}

This study was financed partially by Higher Education Key Laboratory for Measuring and Control Technology and Instrumentation of Heilongjiang Province, Harbin University of Science and Technology.

\section{References}

[1] Y. Jiangtao, "Research on Routing and Scheduling Algorithms for Mobile Ad Hoc Networks", Beijing University of Posts and Telecommunications, (2009), pp. 1-4.

[2] D. Wei and W. Hanggang, "Application of Network Simulation in Network Performance Indicator Evaluation", Computer Engineering and Applications, vol. 18, (2003), pp. 176-180.

[3] B. R. D. Chakeres, "AODV Routing Protocol Implementation Design", 24th International Conference on Distributed Computing Systems Workshops, (2004), pp. 69-70.

[4] S. Wen, W. Bing and Z. Yingbin, "Wireless Sensor Network Technology and Application", Beijing: Electronic Industry Press, (2007), pp. 1-4, 21-26, 65-92.

[5] W. Xiaohong, "Study on Mobile Ad Hoc Network Routing Protocols and Their Performance", Northwestern Polytechnical University, (2005), pp. 27-28.

[6] L. Na, "Study on Mobile Ad Hoc Network Routing Protocols", Hebei University of Science \& Technology, (2010), pp. 36-43.

[7] A. Mareo and J. J. Gareia, "Improving Route Discovery in on-demand Routing Protocols Using Twohop Connected Dominating Sets", Ad Hoc Networks, vol. 4, no. 4, (2006), pp. 509-531.

[8] Y. Chun and S. Meilin, "Research on Ad Hoc Network System Structure", Journal on Communications, vol. 20, no. 9, (1999), pp. 48-49. 